\title{
A Structural Ensemble of a Tau-Microtubule Complex Reveals Regulatory Tau Phosphorylation and Acetylation Mechanisms
}

Z. Faidon Brotzakis, Philip R. Lindstedt, Ross J. Taylor, Dillon J. Rinauro, Nicholas C. T. Gallagher, Gonçalo J. L. Bernardes, and Michele Vendruscolo*

Cite This: ACS Cent. Sci. 2021, 7, 1986-1995

Read Online

ABSTRACT: Tau is a microtubule-associated protein that regulates the stability of microtubules. We use metainference cryoelectron microscopy, an integrative structural biology approach, to determine an ensemble of conformations representing the structure and dynamics of a tau-microtubule complex comprising the entire microtubule-binding region of tau (residues 202-395). We thus identify the ground state of the complex and a series of excited states of lower populations. A comparison of the interactions in these different states reveals positions along the tau sequence that are important to determine the overall stability of the tau-microtubule complex. This analysis leads to the identification of positions where phosphorylation and acetylation events have destabilizing effects, which we validate by using site-specific posttranslationally modified tau variants obtained by chemical mutagenesis. Taken together, these results illustrate how the simultaneous determination of ground and excited states of macromolecular complexes reveals functional and regulatory mechanisms.

\section{INTRODUCTION}

Microtubules are essential components of the cytoskeleton, and they are formed by the polymerization of dimers of $\alpha$ tubulin and $\beta$-tubulin, which are stabilized by a family of microtubule-associated proteins, of which tau is a member. ${ }^{1}$ Tau has micromolar affinity to microtubules, primarily interacting through its microtubule-binding domain (MBD) repeats (R1-R4) and thereafter through the proline-rich domain (Figure 1A). ${ }^{2,3}$ The $\mathrm{C}$-terminal region of $\beta$-tubulin also contributes to the stability of the tau-microtubule complex. ${ }^{4,5}$ Advances in cryoelectron microscopy (cryo-EM) have recently led to the determination of the structure of the $\mathrm{R} 1$ and R2 regions in complex with microtubules (Figure 1A). ${ }^{6}$ Other regions of the tau-microtubule complex were determined at a lower resolution $(0.45-0.65 \mathrm{~nm})$ due to conformational heterogeneity of the corresponding tau and microtubule regions, preventing a fully atomistic description of some of the stabilizing contacts, especially in the R3 and R4 regions, the $\mathrm{C}$-terminal region of $\beta$-tubulin, the flexible PGGG regions, and the proline-rich domain.

It is therefore important to characterize the dynamics of this macromolecular complex in order to better understand the structural basis for the stabilization of microtubules by tau and for the regulatory role of tau phosphorylation and acetylation sites. This goal can be achieved by using cryo-EM (single particle) density maps to determine a structural ensemble representing the conformational heterogeneity of the complex. ${ }^{7}$ The simultaneous determination of the structure and dynamics of proteins is a quantitative approach that enables one to generate an ensemble of structures that recapitulate the information contained in the experimental measurements not only about the conformation of a system but also about its conformational fluctuations. ${ }^{7,8}$ This is possible because bulk experimental measurements, such as those carried out in cryoEM, are averaged in time and space over a large number of individual single particles that populate different conformations. A major challenge toward this goal is to disentangle in the measurements the effects of macromolecular dynamics from those of systematic and random errors in the measurements. The recent development of inference approaches, such as the metainference method, has been able to overcome this challenge. $^{9-13}$ The goal of metainference is to accurately model a structural ensemble by optimally combining experimental data with the prior information available about the system in terms of molecular mechanics. An important aspect of this procedure is that it enables the separation of the effects on the resolution of the electron density maps due to the noise in the experimental input data from the effects resulting from the conformational dynamics of a macro-

Received: May 20, 2021

Published: December 8, 2021 


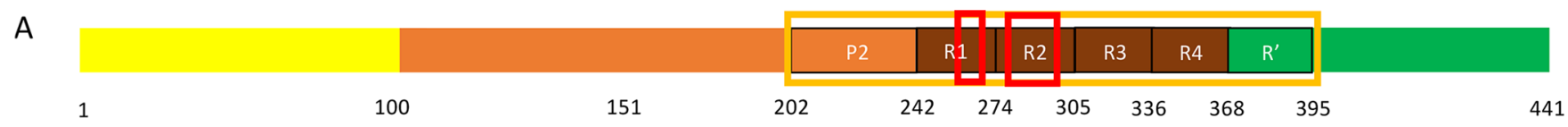

B

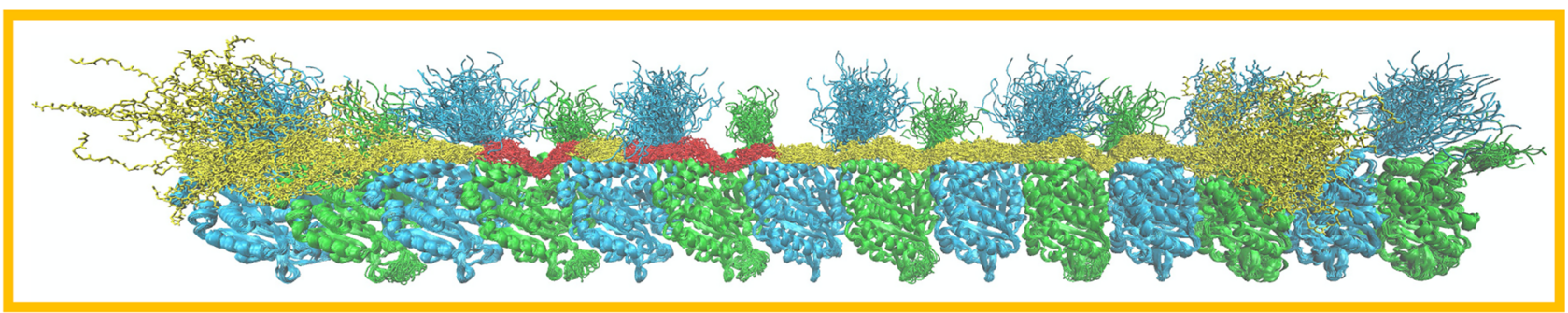

Figure 1. Simultaneous determination of the structure and dynamics of a tau (residues 202-395) in complex with a microtubule. (A) Illustration of the functional domains in the tau sequence, and sequence alignment of microtubule-binding repeats (R1, R2, R3, R4) and flanking regions (P2, R'). The dashed and solid box sequence regions are referred to as weak and strong regions, respectively. The red solid boxes highlight the two regions whose structure has previously been resolved, ${ }^{6}$ and the gold solid box identifies the region whose structure has been determined here (residues 202-395, comprising the P2, R1, R2, R3, R4, and $\mathrm{R}^{\prime}$ regions, PDB 7PQC). (B) Atomic-resolution structural ensemble of the extended microtubule-binding region of tau (residues 202-395) in complex with a microtubule, as determined by EMMI in this study (PDB 7PQC). $\alpha$ tubulin, $\beta$-tubulin, and tau are colored green, blue, and gold, respectively. Microtubules are presented in a cartoon and tau in a licorice representation. The structural determination that we report in this work utilizes an inferential method ${ }^{9-11}$ that enables the determination of a structural ensemble that represents simultaneously the structure and the dynamics of the tau-microtubule complex; the availability of this structural ensemble makes it possible to identify multiple substates ${ }^{7,11}$ (Figure S2).

molecular complex. Metainference has already been used successfully in a series of complex biological problems in combination with cryo-EM and other techniques. ${ }^{9-14}$

By using this approach, we report the determination of a structural ensemble representing the conformational space populated by an extended region of the microtubule-tau complex comprising the whole microtubule-binding region of tau (residues 202-395). This region pertains the entire MTBR, a segment of the proline-rich domain, hereafter abbreviated the P2 region and a segment of the C-terminal region (Figure 1A) . Post-translational modifications are widely believed to dysregulate the binding of protein tau to microtubules, molecular processes most closely linked to the onset and progression of Alzheimer's disease (AD). ${ }^{15-18}$ This dysregulation is associated with an abnormal hyperphosphorylation and hyperacetylation of tau, which in turn decreases the tau-microtubule affinity and leads to aggregation of tau into pathological assemblies known as neurofibrillary tangles. ${ }^{19-21}$ The high-resolution description of the conformational fluctuations of this complex offers the possibility of predicting the role of post-translational modifications of tau on the stability of the complex. We verify our predictions by using a post-translational chemical mutagenesis approach that enables the site-specific modification of tau. ${ }^{22}$

\section{METHODS}

EMMI. The EMMI method ${ }^{10}$ is a recent extension of metainference, ${ }^{9}$ able to incorporate cryo-EM data as restraints in molecular dynamics simulations. In this way, it provides atomistic structural ensembles that maximally agree with the data, by simultaneously determining and taking into account the respective error in the data. EMMI samples the total energy function

$$
\begin{gathered}
E_{\mathrm{EMMI}}=E_{\mathrm{MD}}+k_{\mathrm{B}} T \sum_{r, i} \log \left[\frac{1}{2\left(o v_{\mathrm{DD}, i}-\overline{o v}_{\mathrm{MD}, i}\right)}\right. \\
\left.\quad \operatorname{erf}\left(\frac{o v_{\mathrm{DD}, i}-\overline{o v}_{\mathrm{MD}, i}}{\sqrt{2} \sigma_{r, i}^{\mathrm{SEM}}}\right)\right]
\end{gathered}
$$

where the first term corresponds to the molecular mechanics force field energy, and the second term quantifies an energy penalty that depends on the agreement of the models generated by molecular dynamics with the cryo-EM data. More specifically, EMMI takes as input a Gaussian mixture model (GMM) representation of the voxel cryo-EM map data, henceforth referred to as data GMM. The data GMM comprises $N_{\mathrm{D}}$ components $\phi_{\mathrm{D}, i}$

$$
\phi_{\mathrm{D}}=\sum_{i=1}^{N_{\mathrm{D}}} \phi_{\mathrm{D}, i}(\boldsymbol{x})=\sum_{i=1}^{N_{\mathrm{D}}} \boldsymbol{\omega}_{\mathrm{D}, i} \boldsymbol{G}\left(\boldsymbol{x} \mid \boldsymbol{x}_{\mathbf{D}, i}, \boldsymbol{\Sigma}_{\mathbf{D}, i}\right)
$$

where $\omega_{\mathrm{D}, i}$ is the scaling factor of the $i$ th component of the data GMM, and $G$ is a normalized Gaussian function centered at $\boldsymbol{x}_{\mathrm{D}, i}$ with covariance matrix $\boldsymbol{\Sigma}_{\mathrm{D}, i}$. The agreement between models generated by molecular dynamics and the data GMM is calculated by the following overlap function

$$
o v_{\mathrm{MD}, i}=\int d \boldsymbol{x} \phi_{\mathrm{M}}(\boldsymbol{x}) \phi_{\mathrm{D}, i}(\boldsymbol{x})
$$

where $\phi_{M}(x)$ corresponds to the model GMM, that is a GMM representation of the molecular dynamics atomistic model. In order to deal with the heterogeneity of the system, EMMI simulates many replicas $r$. The overlap between model GMM and data GMM is estimated over the ensemble of replicas to an average overlap per GMM component $\overline{o v}_{\mathrm{MD}, i}$. Finally, $\sigma_{r, i}^{S E M}$ quantifies the error attributed to a finite number of replicas in the simulation one uses to represent the ensemble.

Structural Ensemble Calculations Setup. We start by building a microtubule segment comprising seven $\alpha$-tubulin $/ \beta$ tubulin dimers, obtained from the cryo-EM characterized structure (PDB: 6CVJ). We continue with constructing an initial structure for tau (residues 202-395), where tau- 
microtubule-binding domain regions $\mathrm{R} 1-\mathrm{R} 2$ are based on PDBs 6CVJ and 6CVN. The interactions of R1-R2 with the microtubule are used as a template to build the initial structure of tau region R3-R4 and simultaneously fit the R3-R4 structure in the corresponding density of the full-length tau cryo-EM map (EMD-7522). We achieved this by first fitting a polyalanine model in the Rosetta package ${ }^{23}$ and then adding side chains. Residues 202-241 (P2 region) and 369-395 ( $\mathrm{R}^{\prime}$ region) are built with a random initial structure as these regions are flexible as indicated by the absence of strong density in the tau-microtubule full-length cryo-EM map. In all of the calculations, both tau and the microtubule were considered without post-translational modifications, consistently with the experimental system. ${ }^{6}$

Molecular Dynamics Equilibration. We continued with setting a $9.7 \times 11.4 \times 63.6 \mathrm{~nm}$ simulation box, solvating with 202493 water molecules and neutralizing it by adding ions. We used the AMBER99SB-ILDN ${ }^{24}$ and TIP3P ${ }^{25}$ protein and water force field, respectively. We continued with an energy minimization step followed by a short NPT simulation, followed by a short NVT simulation.

All bonds are constrained with LINCS. ${ }^{26}$ A cutoff value of 1 $\mathrm{nm}$ is used for the Lennard-Jones interactions. The particle mesh Ewald method is used to calculate the electrostatic interactions with a Fourier spacing of $0.12 \mathrm{~nm}$ and a $1 \mathrm{~nm}$ cutoff for the short-range electrostatic interactions. The pair lists are updated every $10 \mathrm{fs}$ with a cutoff of $1 \mathrm{~nm}$ and the time step was $2 \mathrm{fs}^{25}$ A leapfrog algorithm for integrating Newton's equations of motion is used, with a velocity-rescale thermostat $^{27}$ with a coupling time constant of $0.2 \mathrm{ps}$ and a ParrinelloRahman barostat ${ }^{28}$ with a coupling time constant of 1.0 ps for performing an NPT simulation. In the NPT, C $\alpha$ are positionrestrained with a constant $200 \mathrm{~kJ} / \mathrm{mol} \mathrm{nm}{ }^{2}$, the temperature is set to $310 \mathrm{~K}$, the pressure is srt to $1 \mathrm{~atm}$, and the simulation duration is 500 ps. In the NVT simulation, the positionrestraints are lifted, the simulation duration is $2 \mathrm{~ns}$, and the temperature is set to $310 \mathrm{~K}$ without pressure coupling.

EMMI Simulations. First, we expressed the experimental voxel map data as a data GMM containing 47157 Gaussians in total showing a 0.95 correlation to the original voxel experimental map. We continued by extracting 32 configurations from the previous NVT step and initiating two individual EMMI simulations, each consisting of 32 replicas and an aggregate runtime of $400 \mathrm{~ns}$ using PLUMED.2.6.0dev. $^{29}$ EMMI simulations were performed in the NVT ensemble using the same $\mathrm{MD}$ parameters as in the equilibration step. Configurations were saved every 5 ps for postprocessing. The cryo-EM restraint was calculated every 2 $\mathrm{MD}$ steps, using neighbor lists to compute the overlaps between model and data GMMs, with cutoff equal to 0.01 and update frequency of 100 steps. In each EMMI simulation, we excluded the first $2 \mathrm{~ns}$, divided the rest in two segments, and performed cluster analysis using GROMOS ${ }^{30}$ on side-chain and backbone atoms and a $0.3 \mathrm{~nm}$ cutoff. The clustering algorithm counts the number of neighbors using a cutoff, and then it takes the structure with the largest number of neighbors with all its neighbors as a cluster and then eliminates it from the pool of clusters. Each structure of a particular cluster is similar up to a $0.3 \mathrm{~nm}$ cutoff threshold. For convergence purposes, in each EMMI simulation, we calculated cluster population averages and errors based on these two simulation segments. For molecular visualizations, we used $\mathrm{VMD}^{31}$ and Chimera. $^{32}$
Tau Expression and Purification. 2N4R tau lacking the endogenous cysteine residues (C291S and C322S) and the relevant cysteine mutants (S262C and $\mathrm{K} 311 \mathrm{C}$ ), created by standard site-directed mutagenesis, were expressed from a pet29b vector in BL21 Gold (DE3) cells (Agilent Technologies). Cultures were grown to an OD600 of 0.6 and then induced with $0.4 \mathrm{mM}$ IPTG and left to express at $18{ }^{\circ} \mathrm{C}$ overnight. Cells were harvested by centrifugation, resuspended in $50 \mathrm{mM}$ MES ( $\mathrm{pH}$ 6.5), $5 \mathrm{mM}$ DTT, $0.1 \mathrm{mM} \mathrm{PMSF}$, and lysed via sonication ( $1 \mathrm{~min} 30 \mathrm{~s} ; 5 \mathrm{~s}$ on, $10 \mathrm{~s}$ off; $40 \%$ amplitude) on ice. The lysed mixture was centrifuged, and tau was isolated via cation exchange using a Hitrap CaptoS column (GE Healthcare LifeSciences, Little Chalfont, U.K). Fractions containing tau as determined by gel electrophoresis were pooled and precipitated by the addition of $20 \%(\mathrm{w} / \mathrm{v})$ ammonium sulfate on ice overnight. The precipitated protein was pelleted by centrifugation and then resuspended in SSPE buffer containing $5 \mathrm{mM}$ DTT. Pure tau was finally isolated via size exclusion chromatography using a Superdex 200 Increase 10/300 GL column (GE Healthcare LifeSciences, Little Chalfont, U.K.) equilibrated with the aforementioned SSPE buffer; only the purest fractions as assessed by gel were kept for experiments.

Dha Formation. ${ }^{22}$ The tau cysteine mutants were buffer exchanged into $20 \mathrm{mM} \mathrm{NaP}$ buffer $(\mathrm{pH} \mathrm{8)}$ ) via $7 \mathrm{k}$ MWCO Zeba spin desalting columns (Thermo Fisher). A total of 200 $\mu \mathrm{L}$ of $50 \mu \mathrm{M}$ protein aliquots was reacted with $50 \mathrm{~mol}$ equiv of methyl 2,5-dibromopentanoate (Sigma-Aldrich) for $12 \mathrm{~h}$ at 37 ${ }^{\circ} \mathrm{C}$ and shaking at $500 \mathrm{rpm}$. Excess methyl 2,5-dibromopentanoate was removed by passing the reactions through $7 \mathrm{k}$ MWCO Zeba spin desalting columns, and then conversion to Dha was verified via LC-MS (Figures S4-S8).

Final Chemical Mutagenesis. ${ }^{22}$ For the creation of the phosphorylation mimetics, $100 \mu \mathrm{L}$ aliquots of $50 \mu \mathrm{M}$ of S262Dha in $20 \mathrm{mM} \mathrm{NaP}_{\mathrm{i}}$ ( $\mathrm{pH} \mathrm{8)}$ were reacted batchwise (5 min intervals) with 30000 mol equiv of sodium thiophosphate $(\mathrm{pH} 8.0,690 \mathrm{mg} / \mathrm{mL}$ suspension, $5 \times 5000$ equiv $)$. The mixtures were left to react for $8 \mathrm{~h}$ at $37^{\circ} \mathrm{C}$ and shaking at 500 rpm, excess sodium thiosulfate was removed via two $7 \mathrm{k}$ MWCO Zeba spin desalting columns, and reaction completion was verified via LC-MS. For the creation of the acetylated mimetic at $\mathrm{K} 311,100 \mu \mathrm{L}$ aliquots of $50 \mu \mathrm{M}$ K311Dha in 20 $\mathrm{mM} \mathrm{NaP}_{\mathrm{i}}(\mathrm{pH} \mathrm{8})$ were reacted with $1000 \mathrm{~mol}$ equiv of $\mathrm{N}$ acetylcysteamine (Sigma-Aldrich) for $12 \mathrm{~h}$ at $37^{\circ} \mathrm{C}$ and shaking at $500 \mathrm{rpm}$. Excess $N$-acetylcysteamine was removed in the same manner as sodium thiophosphate, and completion was again verified by LC-MS (Figures S4-S8).

Protein LC-MS. Protein liquid chromatography-mass spectrometry (LC-MS) was performed on a Xevo G2-S TOF mass spectrometer coupled to an Acquity UPLC system using an Acquity UPLC BEH300 C4 column (1.7 $\mu \mathrm{m}, 2.1 \mathrm{~mm}$ $\times 50 \mathrm{~mm}$ ). Water with $0.1 \%$ formic acid (solvent A) and $95 \%$ $\mathrm{MeCN}$ and $5 \%$ water with $0.1 \%$ formic acid (solvent $\mathrm{B}$ ) were used as the mobile phase at a flow rate of $0.2 \mathrm{~mL} / \mathrm{min}$. The gradient was programmed as follows: $95 \%$ A for $0.93 \mathrm{~min}$, then a gradient to $100 \%$ B over $4.28 \mathrm{~min}$, then $100 \%$ B for $1.04 \mathrm{~min}$, and then a gradient to $95 \%$ A over $1.04 \mathrm{~min}$. The electrospray source was operated with a capillary voltage of $2.0 \mathrm{kV}$ and a cone voltage of $40 \mathrm{~V}$. Nitrogen was used as the desolvation gas at a total flow of $850 \mathrm{~L} / \mathrm{h}$. Total mass spectra were reconstructed from the ion series using the MaxEnt algorithm preinstalled on MassLynx software (v4.1 from Waters) according to the manufacturer's instructions. 

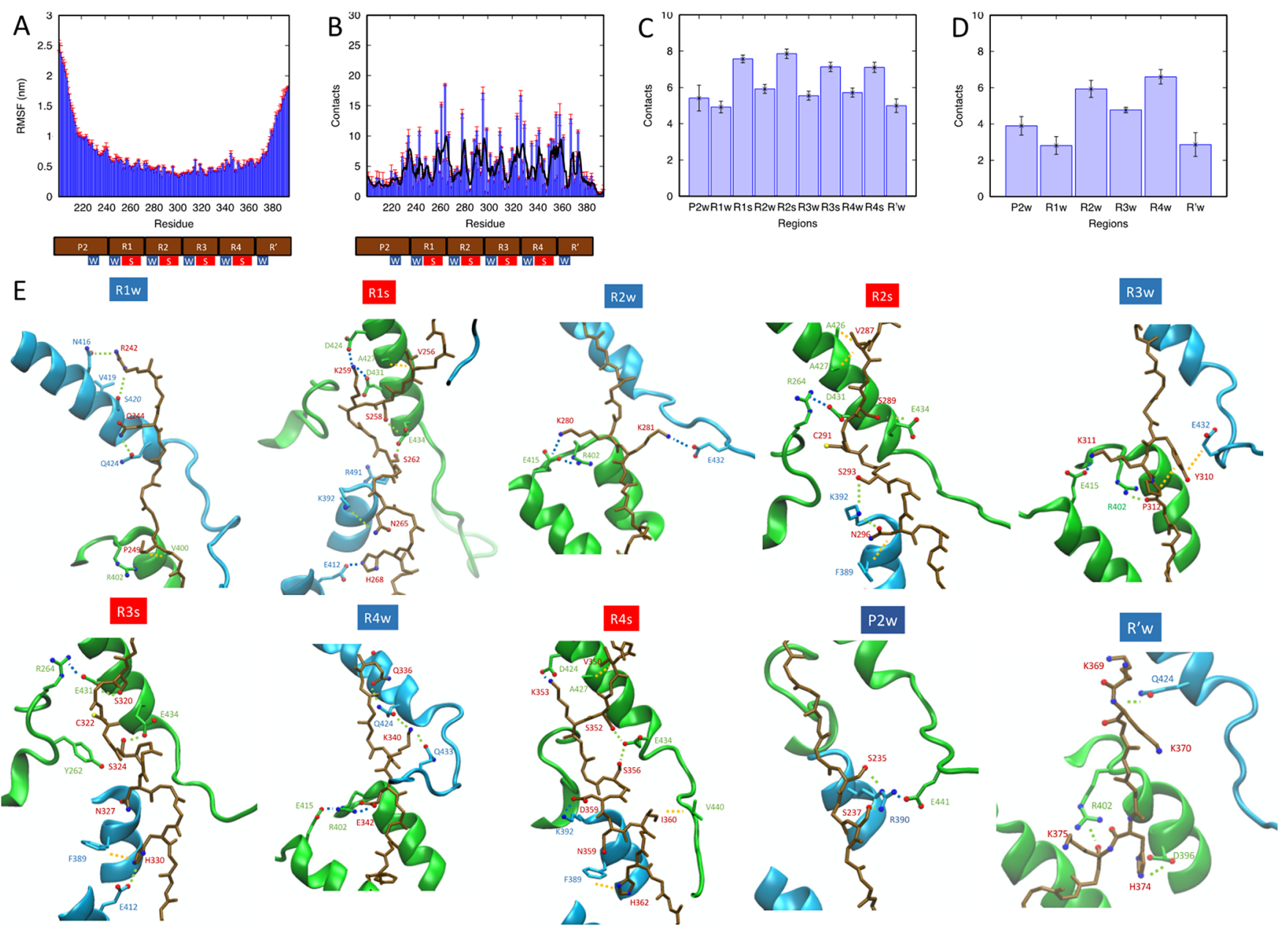

Figure 2. Identification of the stabilizing regions in the tau-microtubule complex. (A) All-atom root-mean-square fluctuations along the tau sequence. (B) Number of contacts between tau residues and microtubules. The black curve indicates a running average over a three-residue window The central residue of the weak [VQI(IN/VY)K] and strong [SK(I/C)GS] interacting regions of tau with microtubules is indicated by blue and solid boxes in the tau sequence panel (in brown). (C) Average number of tau-microtubule contacts per tau region and per residue (normalized by the number of respective residues in the region). (D) Average number of contacts between tau and $\alpha$-tubulin $\beta$-tubulin C-terminal per tau weakly interacting region and per residue (normalized by the number of respective residues in the weak region). (E) Structures of the weakly and strongly interacting regions of the most populated structure as obtained from the clustering analysis. The $\alpha, \beta$-tubulin is colored in green and blue cartoon representations, respectively. Tau is colored in a brown licorice representation. Electrostatic, H-bond, and hydrophobic interactions are highlighted with blue, green, yellow dashed lines, respectively.

Microtubule Polymerization Assay. The microtubule polymerization assays were all performed with reagents from the kit purchased from Cytoskeleton, Inc. (Cat \#BK006P). Tau and the various mutants were buffer exchanged into the tubulin polymerization buffer ( $80 \mathrm{mM}$ PIPES, $2.0 \mathrm{mM} \mathrm{MgCl}$, $0.5 \mathrm{mM}$ EGTA, pH 6.9). GTP was added to the reaction mixture at a final concentration of $1 \mathrm{mM}$. Tubulin aliquots were quickly thawed in a room temperature water bath and immediately added to the samples on ice. Tau and the various chemical mutants were assayed at $15 \mu \mathrm{M}$ for their ability to polymerize tubulin at a concentration of $3 \mathrm{mg} / \mathrm{mL}$. After briefly mixing, samples were directly aliquoted into a prewarmed 96-well half area plate (Corning \#3697). The reaction was monitored by OD at $340 \mathrm{nM}$ using a CLARIOstar Plus plate reader at $37^{\circ} \mathrm{C}$ (BMG Labtech).

\section{RESULTS}

Simultaneous Determination of the Structure and Dynamics of a Tau-Microtubule Complex. The taumicrotubule structural ensemble determined in this study
(Figure 1B) provides insight into the behavior of the microtubule-binding region of tau (in particular, of the previously undetermined regions $\mathrm{P} 2, \mathrm{R} 3, \mathrm{R} 4$, and $\mathrm{R}^{\prime}$ ) and of the $\beta$-tubulin C-terminal region. Notably, the P2 and $\mathrm{R}^{\prime}$ regions of tau and the $\mathrm{C}$-termini of $\alpha, \beta$-tubulin are rather disordered, consistent with the weak densities that these regions have in the cryo-EM maps and NMR experiments. ${ }^{3,6}$ For strong electron density, most of the tau-microtubule structure is identifiable through the metainference cryo-EM (EMMI) method, indicating low levels of structural heterogeneity and a fairly rigid tau-microtubule structure. However, structural ensembles corresponding to the regions of weaker electron density exhibit a higher degree of structural heterogeneity, such as in the case of the R1-R4, P2 and $\mathrm{R}^{\prime}$ regions as well as the $\mathrm{C}$-termini of $\alpha, \beta$-tubulin (Figure S1).

The dynamics of the tau-microtubule complex results in the population of different states. Our results indicate that the complex populates a ground state, as well as several excited states with lower populations (Figure S2), which we obtained 
by performing a clustering analysis of the structural ensemble (Figure 1B).

Stabilizing Regions in the Tau-Microtubule Complex. The tau-microtubule complex exhibits highly dynamical regions characterized by large conformational fluctuations (Figure 2A). In the complex, tau exhibits a rigid microtubulebinding domain region and two flexible flanking regions (P2 and $\left.\mathrm{R}^{\prime}\right)$, with the $\mathrm{P} 2$ region exhibiting greater structural heterogeneity $(4.33 \pm 0.05 \mathrm{~nm})$ than $\mathrm{R}^{\prime}(3.20 \pm 0.04 \mathrm{~nm})$. We also note that region $\mathrm{R} 1$ exhibits slightly greater structural heterogeneity $(0.56 \pm 0.02 \mathrm{~nm})$ than $\mathrm{R} 2(0.43 \pm 0.02 \mathrm{~nm})$, as well as than R3 $(0.42 \pm 0.01 \mathrm{~nm})$ and $\mathrm{R} 4(0.50 \pm 0.02 \mathrm{~nm})$. These values are consistent with the original cryo-EM study ${ }^{6}$ where the resolution of the data in regions $\mathrm{R} 1-\mathrm{R} 4$ spans $0.45-$ $0.6 \mathrm{~nm}$. Such evidence suggests the possibility of an asymmetric unzipping mechanism, where tau preferentially unbinds from microtubules from the $\mathrm{P} 2$ region, that is, from the more unstable + end of the microtubule. Note that direction of dynein motion is also from the + to the - end of the microtubule. We leave this hypothesis to be tested in future simulations.

To further shed light on the interactions that stabilize the tau-microtubule complex, we highlight the tau-microtubule interaction energies quantified by the contacts each tau residue forms with the microtubule (Figure 2B). We thus identify weak and strong interacting regions of tau with microtubules, indicated by dashed and solid arrows, respectively. For each repeat, we identify weakly (R1w, R2w, R3w, and R4w) and strongly (R1s, R2s, R3s, and R4s) interacting regions, which correspond to residue sequences indicated by the dashed and solid boxes in Figure S3A, respectively. The strongly interacting regions of the repeats include the hallmark SK(I/ C) GS motif, known to contribute to tau-microtubule stability and to be associated with $\mathrm{AD}$-related phosphorylation sites. ${ }^{21}$ Notably, the weakly interacting regions also show some sequence conservation motif across the repeats with the PHF6 and PHF6* aggregation-prone VQI(IN/VY)K hallmark motif $^{33}$ present in the $\mathrm{R} 2$ and $\mathrm{R} 3$ regions. As described below, lysine residues in the weak regions play a critical role in stabilizing the tau-microtubule complex. Residues 230-240 (P2w) and 370-379 ( $\left.\mathrm{R}^{\prime} \mathrm{w}\right)$ form weak interactions with the microtubule, with serine residues in the $\mathrm{P} 2 \mathrm{w}$ region and lysine residues in the $R^{\prime} w$ region contributing to the tau-microtubule interactions.

Residues in the weakly and strongly interacting regions form on average about $5-6$ or $7.5-8$ contacts with microtubules, respectively (Figure 2C), with a repetitive pattern of weak interactions followed by strong interactions in the microtubulebinding domain region. Notably, the weakly interaction region of R1 (R1w) forms slightly fewer contacts with the microtubules than the other weak MTBR regions ( $R 2 w$, $\mathrm{R} 3 \mathrm{w}, \mathrm{R} 4 \mathrm{w}$ ), as shown in Figure 2C. These observations are attributed to the fewer interactions that the $\mathrm{R} 1 \mathrm{w}$ region makes with the $\mathrm{C}$-terminus of $\beta$-tubulin as opposed to the $\mathrm{R} 2 \mathrm{w}, \mathrm{R} 3 \mathrm{w}$, and $\mathrm{R} 4 \mathrm{w}$ regions (Figure 2D) and can explain the higher flexibility of the R1 region with respect to the rest of the repeat regions. Thus, the $\beta$-tubulin $\mathrm{C}$-terminus stabilizes the taumicrotubule complex by forming interactions with the weak regions and could explain the stabilizing role of $\mathrm{C}$-termini in tau-microtubule complex. ${ }^{4,5}$

Interatomic Interactions Stabilizing the Tau-Microtubule Complex. Tau interacts in a similar manner with tubulin along the strongly interacting regions (R1s, R2s, R3s, and R4s), in particular, with $\alpha$-helix H12 of $\alpha$-tubulin and $\alpha$ helices $\mathrm{H} 11$ and $\mathrm{H} 12$ of $\beta$-tubulin, by hydrogen bonds ( $\mathrm{H}-$ bonds), electrostatic and hydrophobic interactions (Figure $2 \mathrm{E}$ ). The weakly interacting regions ( $\mathrm{R} 1 \mathrm{w}, \mathrm{R} 2 \mathrm{w}, \mathrm{R} 3 \mathrm{w}, \mathrm{R} 4 \mathrm{w}$, and $\mathrm{R}^{\prime} \mathrm{w}$ ) also interact in a similar manner along the repeat sequence, namely, with $\alpha$-helix H12 of $\beta$-tubulin, and $\alpha$-helices $\mathrm{H} 11$ and $\mathrm{H} 12$ of $\alpha$-tubulin by hydrogen bonds (H-bonds), electrostatic and hydrophobic interactions. The only exception to this interaction symmetry is the $\mathrm{P} 2 \mathrm{w}$ region, which interacts with parts of tubulin that a strong interacting region would be engaging with, i.e., with $\alpha$-helix H12 of $\alpha$-tubulin as well as with $\alpha$-helices $\mathrm{H} 11$ and $\mathrm{H} 12$ of $\beta$-tubulin. This is an obligatory action for tau since the entire SK(I/C)GS...PGGG sequence domain existing in the microtubule-binding repeats is missing in the P2 region (Figure S3A), and therefore residues in the weakly interacting regions replace such interactions. This mismatch thus leads to weaker interactions of the P2 region with microtubules.

The strongly interacting regions (R1s, R2s, R3s, R4s) are characterized by a conserved sequence identity (Figure S3A), as well as conserved interaction between amino acids of tau and tubulin, from repeat to repeat. In particular V256, V287, V316, and V350 make hydrophobic interactions with A427 in $\alpha$-tubulin (Figure 2E). The residues of the SK(I/C)GS motif interact as follows with microtubules: residues S258, S262, S289, S320, S324, S352, and S356 form hydrogen bonds with residues E434 of $\alpha$-tubulin, apart from S293 and S320 that form alternative hydrogen bonds with K392 and E431 respectively (Figure 2E). Residues K259, K353 form electrostatic interactions with residues D424, D431 (Figure 2E). In addition, tau residues N265, N296, N327, and N359 form hydrogen bond and hydrophobic interactions with $\beta$-tubulin K392 and D389 respectively (Figure 2E). Finally, conserved tau residues $\mathrm{H} 268, \mathrm{H} 330$, and $\mathrm{H} 362$ form hydrogen bond and hydrophobic interactions with $\beta$-tubulin E412 and F389 respectively (Figure $2 \mathrm{E}$ ). Taken together, our structures give an atomistic insight into these stabilizing interactions and can structurally explain the impairment of tau assembly upon pseudophosphorylation or pseudocetylation of S262, S324, S356, K259, and K353 found in vitro experiments. ${ }^{21,34,35}$

In the weakly interaction region $\mathrm{R} 1 \mathrm{w}$, residue $\mathrm{R} 242$ of tau forms hydrogen bonds with residues N416 and S420 of $\alpha$ tubulin, and residue Q244 and P249 of tau forms hydrogen bonds and hydrophobic interactions with $\alpha$-tubulin Q424 and $\beta$-tubulin V400, respectively (Figure $2 \mathrm{E}$ ). In the weakly interacting region $\mathrm{R} 2 \mathrm{w}$, residues $\mathrm{K} 280$ and $\mathrm{K} 281$ of tau form electrostatic interactions with $\alpha$-tubulin residues R402, E414 and the $\mathrm{C}$-terminal residue $\mathrm{E} 432$ of $\beta$-tubulin. In the weakly interacting region $\mathrm{R} 3 \mathrm{w}$, residues $\mathrm{Y} 310, \mathrm{~K} 311$ and $\mathrm{P} 312$ of tau form a combination of electrostatic and hydrophobic interactions with $\alpha$-tubulin R402, E415 and C-terminal $\beta$ tubulin residue E432 respectively (Figure 2E). Notably, residues $\mathrm{K} 280, \mathrm{~K} 281, \mathrm{Y} 310$, and $\mathrm{K} 311$ are part of the $\mathrm{VQI}(\mathrm{IN} / \mathrm{VY}) \mathrm{K}$ sequence preserved motif and residues K280, $\mathrm{K} 281$ are known to contribute to the stability of the taumicrotubule complex, while the entire motif is an aggregationprone motif. ${ }^{33,35}$ The $\mathrm{R} 4 \mathrm{w}$ region comprises notable hydrogen bond and electrostatic interactions between residues Q336, $\mathrm{K} 340$, and E342 of tau with residues Q424, Q433 of $\beta$-tubulin and $\mathrm{R} 402, \mathrm{E} 415$ of $\alpha$-tubulin (Figure 2E). The $\mathrm{R}^{\prime} \mathrm{w}$ region forms notable hydrogen bond interactions between residues tau K369, K370, K375, and H374 with residues Q424 of $\beta$ tubulin and D396, R402 of $\alpha$-tubulin. Finally, in the P2w 

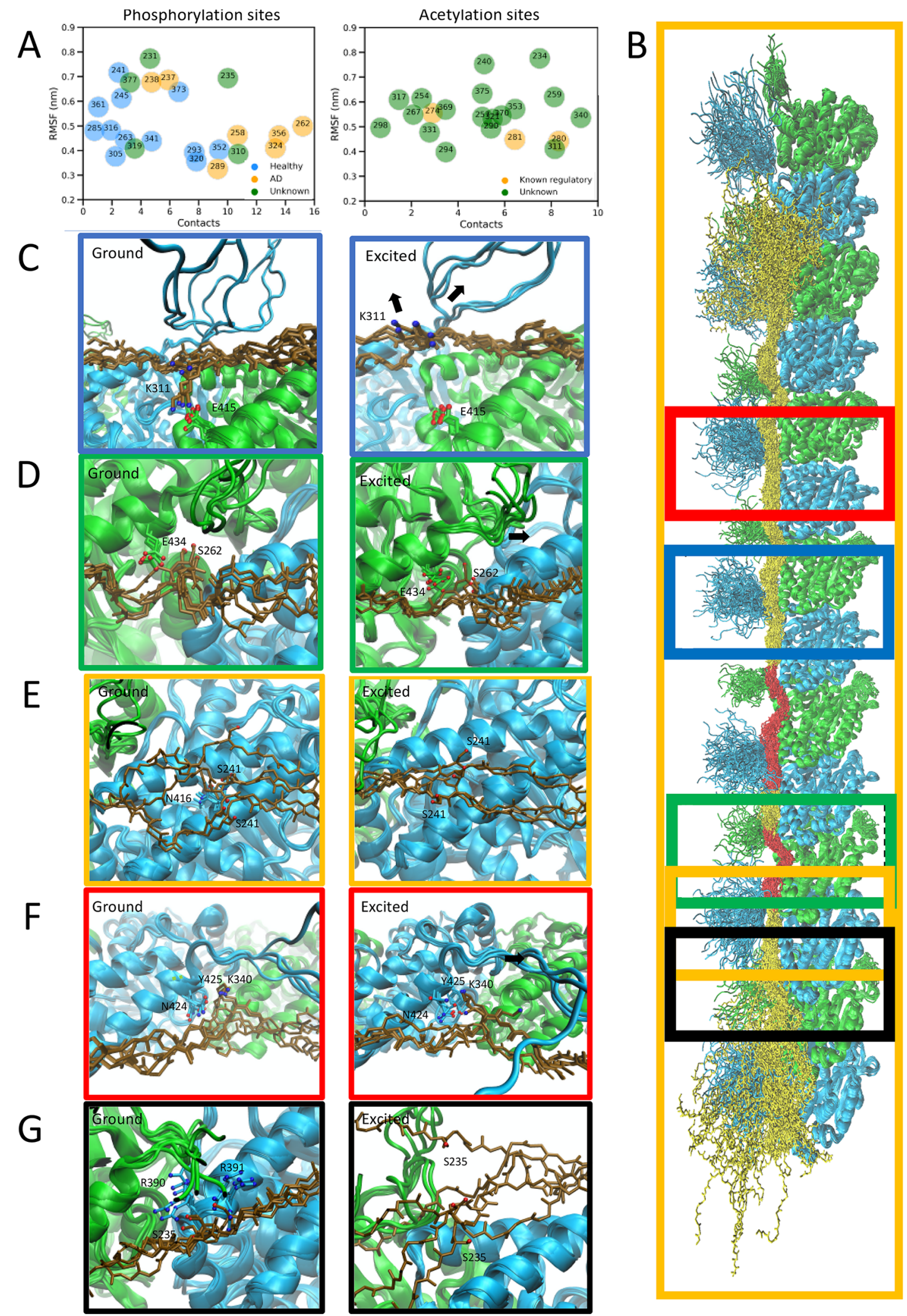

Figure 3. Identification of phosphorylation and acetylation sites altering the stability of the tau-microtubule complex. (A) By considering the contributions to the stability of the tau-microtubule complex (number of contacts of a residue, $x$-axis) and the accessibility to kinases (structural heterogeneity, RMSF, $y$-axis), we can identify the residues in the upper right region of the plot as those that are expected to be most important for stability and that have the higher propensity to be phosphorylated (left panel) or acetylated (right panel). Our results show that most residues known to be phosphorylated in $\mathrm{AD}$ (in orange) are of this type. Residues phosphorylated in healthy brains (in blue) tend instead to have fewer contacts and therefore to have a weaker effect on the stability. From this analysis, we identify S262 as potentially having a significant effect on the stability. Phosphorylation data are obtained from ref 21 . Similarly, we can identify residues expected to be most important for stability and have the higher propensity to be acetylated. Our results show that two of the residues with regulatory function, known to be acetylated in $\mathrm{AD}$ (K280 and 
Figure 3. continued

$\mathrm{K} 281$, in orange), are of this type. Among the residues whose acetylation state is not known (in green), we identify K311 as potentially having a significant effect on the stability. Acetylated data are obtained from refs 17 and 18. (B) Regions of residues S235 (black), S241 (gold), S262 (green) and K311 (blue), K340 (red), which we identified for post-translational modifications. Panels C-G indicate the ground and excited state structural ensembles of the regions of K340, K311, S262, S241, and S235. Details of the structural ensemble show interactions of tau K340 with $\beta$-tubulin Q424 and C-terminal (C), of tau K311 with $\alpha$-tubulin E415 and $\beta$-tubulin C-terminal (D), of tau S262 and tubulin E434 and $\alpha$-tubulin C-terminal (E), of tau S241 and tubulin N416 (F), and of tau S235 and $\beta$-tubulin R390-R391 and $\alpha$-tubulin C-terminal (G). Tau is highlighted in brown licorice, $\beta$-tubulin in blue, and $\alpha$-tubulin in green. The arrows indicate the motion of the tubulin $\mathrm{C}$-termini of $\alpha$, $\beta$-tubulin as well as the breakage of K311 interactions with E415 in the excited state with respect to the ground state. The comparison between the structures of the ground states and the excited states illustrates the availability in the excited state of the sites for post-translational modifications.

region, residues S235, S237 of tau interact with residues E441 of $\alpha$-tubulin and R390 of $\beta$-tubulin (Figure 2E).

These results are in agreement with previously determined structures of the R1 and R2 regions ${ }^{6}$ (Figure S3B,C) with an all-atom RMSD of $0.27 \pm 0.15 \mathrm{~nm}$ for the $\mathrm{R} 1$ region and $0.3 \pm$ $0.2 \mathrm{~nm}$ for the $\mathrm{R} 2$ region from different structures of the most populated cluster of our EMMI ensemble.

Identification and Validation of Phosphorylation Sites Altering the Stability of the Tau-Microtubule Complex. The phosphorylation of tau can lead to a loss of stabilizing tau-microtubule interactions, molecular processes most closely linked to the onset and progression of Alzheimer's disease $(\mathrm{AD}) .^{15-18}$ The knowledge of the structure and dynamics of the tau-microtubule complex enables a connection to be made between the strength of the tau-microtubule interactions and the propensity of residues to be phosphorylated. Such a connection is not limited to only verify existing phosphorylation sites altering the tau-microtubule stability but also to predict new ones. We analyzed the average number of contacts and the conformational heterogeneity of serine, threonine, and tyrosine residues, which are possible sites of phosphorylation (Figure 3A). According to this metric, residues that contribute most to the stability are those that form a high number of contacts. One could then speculate that residues that have high conformational heterogeneity may have a higher chance to become accessible to kinases and be posttranslationally modified at the tau-microtubule interface. ${ }^{36} \mathrm{We}$ investigated this hypothesis by identifying known tau-microtubule stability-altering phosphorylation sites in $\mathrm{AD}$ brains (Figure 3A, orange sites), including S258, S262, S324, and S356, ${ }^{19,20,34}$ since they form many contacts with microtubules and have a moderate flexibility. In addition, this analysis predicts known phosphorylated sites in $\mathrm{AD}$ brains with an unknown tau-microtubule stability role, including S237 (P2w) and S289 (R2s) (Figure 3B, orange sites). ${ }^{21}$

Next, we analyzed in more detail the predicted phosphorylation sites at positions S235, S241, and S262 (Figure 3B, black, yellow, and green boxes, respectively). The excited state shows that the S235 and S262 positions become more exposed upon the $\alpha$-tubulin $\mathrm{C}$-terminal motion away from tau and hence possibly more accessible to kinases for phosphorylation (Figure 3C, green box). In contrast, while position S241 remains accessible in both the ground and excited states, it does not form stabilizing contacts (Figure $3 \mathrm{E}$ ); thus, while phosphorylation by kinase activity is feasible, phosphorylation does not significantly perturb tau-microtubule stability, as shown by Figure 4B,C. In order to obtain site-specific phosphorylation at these three sites, we use a chemical mutagenesis approach to mimic phosphorylation. ${ }^{37,38}$ This approach relies on the installation of the amino acid dehydroalanine (Dha) at the site of interest and then a subsequent Michael addition by thiophosphate to install a highly accurate phosphorylation mimic ${ }^{22}$ (Figures S4, S6, and S7). We validate these calculations by a microtubule polymerization assay (Figure 4A,B), which shows that phosphorylation of both S235 and S262 reduces the microtubule polymerization rate, while the phosphorylation of S241 shows no significant effect. Importantly, we also identified sites with ambiguous phosphorylation states between $\mathrm{AD}$ and control brains and unknown tau-microtubule stability role such as T231 (P2w) and Y310 (R2w) (Figure 3A, green sites). ${ }^{21}$ Finally, we observe that phosphorylated residues in healthy brains do not contribute much to the stability of the tau-microtubule complex since they mostly form few interactions with microtubules (Figure 3A, blue sites).

Identification and Validation of Acetylation Sites Altering the Stability of the Tau-Microtubule Complex. Tau acetylation in relation to tau-microtubule stability dysregulation is less studied than phosphorylation. Yet, recent studies show that acetylation can lead to a loss of stabilizing tau-microtubule interactions, molecular processes most closely linked to the onset and progression of Alzheimer's disease (AD). ${ }^{18,35}$ In a similar manner as above, based on the analysis of the structural ensembles, we selected sites expected to alter the tau-microtubule stability upon acetylation; in particular, these sites form many contacts and exhibit moderate to high flexibility (Figure 3A). This analysis identifies the K280, K281, and K274 known sites, which upon acetylation significantly dysregulates the complex stability (Figure $3 \mathrm{~A}$, orange sites), with the first two being related to $\mathrm{AD}$ pathology. ${ }^{35}$ We then also predict K234 (P2w), K240 (P2w), K259 (R1s), K290 (R2s), K311 (R3w), K321 (R3s), K340 (R4w), K353 (R4s), $\mathrm{K} 370\left(\mathrm{R}^{\prime} \mathrm{w}\right), \mathrm{K} 375\left(\mathrm{R}^{\prime} \mathrm{w}\right)$ as affinity-regulating acetylation sites (Figure 3A, green sites). K259, K290, K321, and K353 belong to the KXGS domain, while stronger contacts such as K280 $(\mathrm{R} 2 \mathrm{w}), \mathrm{K} 311(\mathrm{R} 3 \mathrm{w})$, and $\mathrm{K} 340(\mathrm{R} 4 \mathrm{w})$ are repeated lysine residues on the weak interaction region of tau VQI(IN/VY)K. Next, we analyzed in more detail the acetylation at positions K311 and K340. The excited state shows that position K311 becomes more exposed by losing contacts with the $\beta$-tubulin C-terminal and $\alpha$-tubulin E415 and hence possibly more accessible to acetylation enzymes (Figure 3C, blue box). The local environment at position K340 appears to be highly flexible (Figure 3F, red box), thereby more solvated in the excited state than the ground state. Thus, K340 is presumably more susceptible to acetyltransferase activity. In order to obtain site-specific acetylation at K311 and K340, we used the same chemical mutagenesis approach as taken with the phosphorylation mimetics except that the Michael addition to Dha at these two sites was instead carried out by $N$ acetylcysteamine (Figures S5 and S8). 


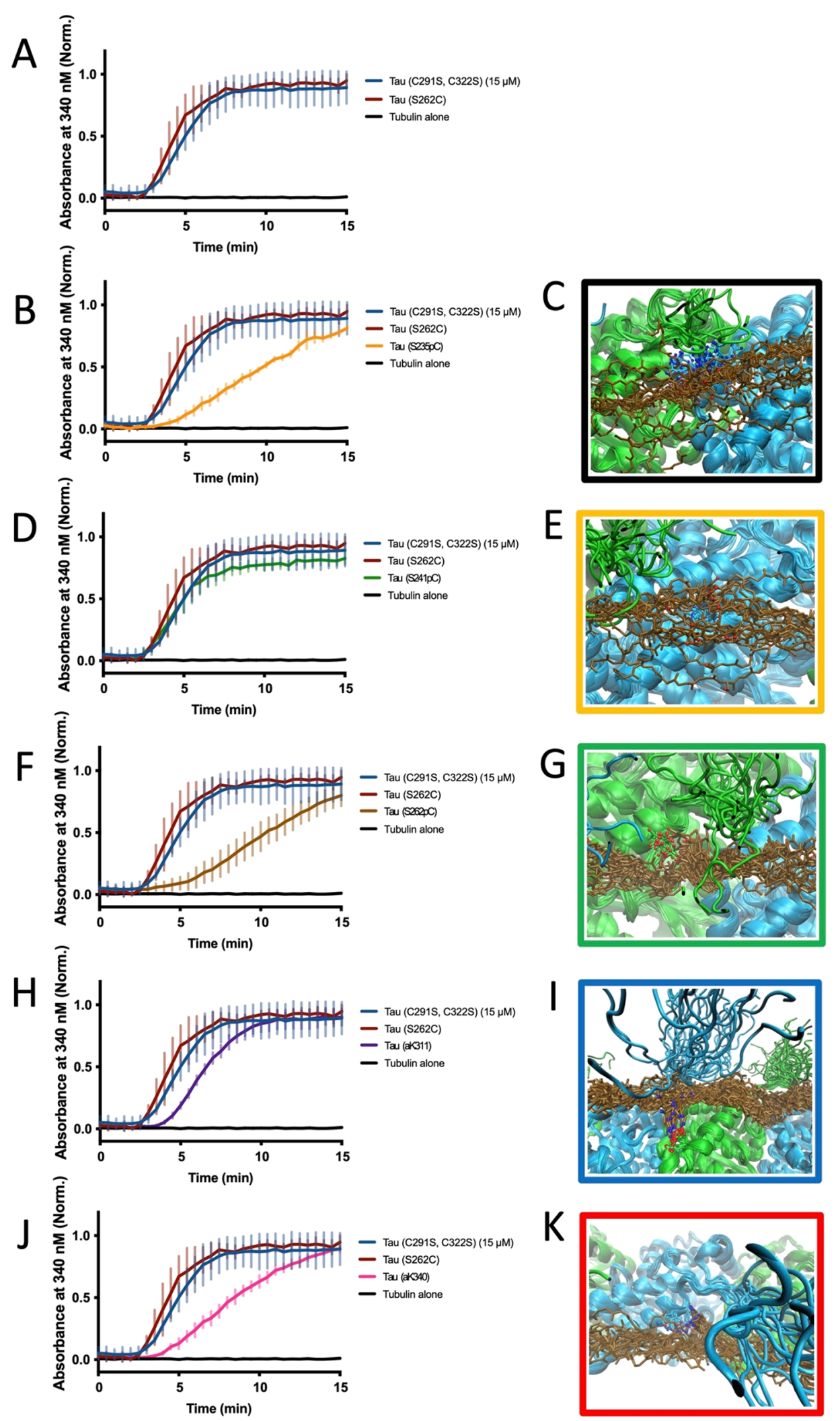

Figure 4. Validation of phosphorylation and acetylation sites altering the stability of the tau-microtubule complex. (A) Tubulin polymerization assay in the presence and absence of the tau variants used as a starting point for chemical mutagenesis in this work. (B, C) Validation of the effect of phosphorylation of S235 on tubulin polymerization (B) and conformational heterogeneity of the interaction of tau S235 with tubulin R390, R391 (C). (D, E) Validation of the effect of phosphorylation of S241 on tubulin polymerization (D) and conformational heterogeneity of the interaction of tau S241 with tubulin (E). (F, G) Validation of the effect of phosphorylation of S262 on tubulin polymerization (F) and conformational heterogeneity of the interaction of tau S262 with E434 and $\alpha$-tubulin C-terminal (G). (H, I) Validation of the effect of acetylation of K311 on tubulin polymerization $(\mathrm{H})$ and conformational heterogeneity of the interaction of tau K311 with tubulin E415 (I). (J, K) Validation of the effect of acetylation of K340 on tubulin polymerization (J) and conformational heterogeneity of the interaction of tau K340 with tubulin Q424. 
We then validated these predictions by a microtubule polymerization assay (Figure $4 \mathrm{C}, \mathrm{D}$ ), which shows that both acetylation of $\mathrm{K} 311$ and $\mathrm{K} 340$ reduce the microtubule polymerization rate, hence having an impact for $\mathrm{AD}$ by possibly promoting NFT formation.

\section{CONCLUSIONS}

We have reported a structural ensemble of an extended microtubule-binding region of tau (residues 202-395) in a complex with a microtubule. The results that we have presented reveal how the conformational fluctuations in the complex lead to the population of excited states. We then identified post-translational modification sites that take part in the regulation of the binding of tau to microtubules and validated their effects on microtubule polymerization through a site-specific chemical mutagenesis approach. These results provide a mechanistic understanding of molecular processes closely linked to the progression of Alzheimer's disease. $^{15,16,21,35}$

\section{ASSOCIATED CONTENT}

\section{SI Supporting Information}

The Supporting Information is available free of charge at https://pubs.acs.org/doi/10.1021/acscentsci.1c00585.

Back-calculated electron density maps from the EMMI ensemble of the tau-microtubule complex; identification of the ground and excited states of the R1-R4, P2, and $\mathrm{R}^{\prime}$ regions of tau in the EMMI structural ensemble; sequence alignment of the tau regions P2, R1, R2, R3, $\mathrm{R} 4$, and $\mathrm{R}^{\prime}$. LC-MS spectra of tau and its phosphorylated chemical mutant at S262 and its intermediates; LC-MS spectra of the acetylated K311 (aK311) tau chemical mutant and its intermediates; LC-MS spectra of the phosphorylated chemical mutant of tau at S235 and its intermediates; LC-MS spectra of the phosphorylated chemical mutant of tau at S241 and its intermediates (PDF)

\section{Accession Codes}

We deposited in the protein data bank (PDB) a representative example of a structure in the ensemble of the tau-microtubule complex (PDB 7PQC). For comparison, we have also deposited in the PDB a structure of the excited state of region R4 indicated in Figure 3C (PDB 7PQP).

\section{AUTHOR INFORMATION}

\section{Corresponding Author}

Michele Vendruscolo - Centre for Misfolding Diseases, Department of Chemistry, University of Cambridge, Cambridge CB2 1EW, U.K.; 10 orcid.org/0000-0002-36161610; Email:mv245@cam.ac.uk

\section{Authors}

Z. Faidon Brotzakis - Centre for Misfolding Diseases, Department of Chemistry, University of Cambridge, Cambridge CB2 1EW, U.K.

Philip R. Lindstedt - Centre for Misfolding Diseases, Department of Chemistry, University of Cambridge, Cambridge CB2 1EW, U.K.

Ross J. Taylor - Centre for Misfolding Diseases, Department of Chemistry, University of Cambridge, Cambridge CB2 $1 E W$, U.K.
Dillon J. Rinauro - Centre for Misfolding Diseases, Department of Chemistry, University of Cambridge, Cambridge CB2 1EW, U.K.

Nicholas C. T. Gallagher - Centre for Misfolding Diseases, Department of Chemistry, University of Cambridge, Cambridge CB2 1EW, U.K.

Gonçalo J. L. Bernardes - Centre for Misfolding Diseases, Department of Chemistry, University of Cambridge, Cambridge CB2 1EW, U.K.; Instituto de Medicina Molecular João Lobo Antunes, Faculdade de Medicina Universidade de Lisboa, 1649-028 Lisboa, Portugal; 이이이.org/00000001-6594-8917

Complete contact information is available at:

https://pubs.acs.org/10.1021/acscentsci.1c00585

\section{Notes}

The authors declare no competing financial interest.

EMMI input, system topology, and structure files can be found in PLUMED-NEST (plumID: 21.040) https://www.plumednest.org/eggs/21/040/. GROMACS files and raw trajectory data can be found in the github repository https://github. $\mathrm{com} /$ vendruscolo-lab/tau-microtubules and in the zenobo repository https://zenodo.org/record/5507453\#. YUINRi0RrJE

\section{ACKNOWLEDGMENTS}

The authors would like to acknowledge Dr. Max Bonomi, Dr. Liz Kellogg, and Dr. Eva Nogales for discussions, and the ARCHER supercomputer system for making computer time available. Z.F.B. would like to acknowledge the Federation of European Biochemical Societies (FEBS) for financial support through a long-term fellowship. R.J.T. is supported by the Biotechnology and Biological Sciences Research Council (BBSRC; BB/M011194), and G.J.L.B. is a Royal Society University Research Fellow (URF/R/180019).

\section{REFERENCES}

(1) Conde, C.; Cáceres, A. Microtubule assembly, organization and dynamics in axons and dendrites. Nat. Rev. Neurosci. 2009, 10 (5), 319-332.

(2) Kar, S.; Fan, J.; Smith, M. J.; Goedert, M.; Amos, L. A. Repeat motifs of tau bind to the insides of microtubules in the absence of taxol. ЕMBO J. 2003, 22 (1), 70-77.

(3) Kadavath, H.; Jaremko, M.; Jaremko, Ł.; Biernat, J.; Mandelkow, E.; Zweckstetter, M. Folding of the tau protein on microtubules. Angew. Chem., Int. Ed. 2015, 54 (35), 10347-10351.

(4) Chau, M.-F.; Radeke, M. J.; de Inés, C.; Barasoain, I.; Kohlstaedt, L. A.; Feinstein, S. C. The microtubule-associated protein tau crosslinks to two distinct sites on each $\alpha$ and $\beta$ tubulin monomer via separate domains. Biochemistry 1998, 37 (51), 17692-17703.

(5) Serrano, L.; Montejo De Garcini, E.; Hernandez, M. A.; Avila, J. Localization of the tubulin binding site for tau protein. Eur. J. Biochem. 1985, 153 (3), 595-600.

(6) Kellogg, E. H.; Hejab, N. M.; Poepsel, S.; Downing, K. H.; DiMaio, F.; Nogales, E. Near-atomic model of microtubule-tau interactions. Science 2018, 360 (6394), 1242-1246.

(7) Bonomi, M.; Heller, G. T.; Camilloni, C.; Vendruscolo, M. Principles of protein structural ensemble determination. Curr. Opin. Struct. Biol. 2017, 42, 106-116.

(8) Lindorff-Larsen, K.; Best, R. B.; DePristo, M. A.; Dobson, C. M.; Vendruscolo, M. Simultaneous determination of protein structure and dynamics. Nature 2005, 433 (7022), 128-132.

(9) Bonomi, M.; Camilloni, C.; Cavalli, A.; Vendruscolo, M. Metainference: A Bayesian inference method for heterogeneous systems. Sci. Adv. 2016, 2 (1), No. e1501177. 
(10) Bonomi, M.; Pellarin, R.; Vendruscolo, M. Simultaneous determination of protein structure and dynamics using cryo-electron microscopy. Biophys. J. 2018, 114 (7), 1604-1613.

(11) Bonomi, M.; Vendruscolo, M. Determination of protein structural ensembles using cryo-electron microscopy. Curr. Opin. Struct. Biol. 2019, 56, 37-45.

(12) Eshun-Wilson, L.; Zhang, R.; Portran, D.; Nachury, M. V.; Toso, D. B.; Löhr, T.; Vendruscolo, M.; Bonomi, M.; Fraser, J. S.; Nogales, E. Effects of $\alpha$-tubulin acetylation on microtubule structure and stability. Proc. Natl. Acad. Sci. U. S. A. 2019, 116 (21), 1036610371.

(13) Vahidi, S.; Ripstein, Z. A.; Bonomi, M.; Yuwen, T.; Mabanglo, M. F.; Juravsky, J. B.; Rizzolo, K.; Velyvis, A.; Houry, W. A.; Vendruscolo, M.; Rubinstein, J. L.; Kay, L. E. Reversible inhibition of the ClpP protease via an $\mathrm{N}$-terminal conformational switch. Proc. Natl. Acad. Sci. U. S. A. 2018, 115 (28), E6447-E6456.

(14) Brotzakis, Z. F.; Lohr, T.; Vendruscolo, M. Determination of intermediate state structures in the opening pathway of SARS-CoV-2 spike using cryo-electron microscopy. Chem. Sci. 2021, 12, 9168.

(15) Dujardin, S.; Commins, C.; Lathuiliere, A.; Beerepoot, P.; Fernandes, A. R.; Kamath, T. V.; Mark, B.; Klickstein, N.; Corjuc, D. L.; Corjuc, B. T. Tau molecular diversity contributes to clinical heterogeneity in Alzheimer's disease. Nat. Med. 2020, 26 (8), 12561263.

(16) Wesseling, H.; Mair, W.; Kumar, M.; Schlaffner, C. N.; Tang, S.; Beerepoot, P.; Fatou, B.; Guise, A. J.; Cheng, L.; Takeda, S.; Muntel, J.; Rotunno, M. S.; Dujardin, S.; Davies, P.; Kosik, K. S.; Miller, B. L.; Berretta, S.; Hedreen, J. C.; Grinberg, L. T.; Seeley, W. W.; Hyman, B. T.; Steen, H.; Steen, J. A. Tau PTM Profiles Identify Patient Heterogeneity and Stages of Alzheimer's Disease. Cell 2020, 183, 1699-1713.

(17) Min, S.-W.; Cho, S.-H.; Zhou, Y.; Schroeder, S.; Haroutunian, V.; Seeley, W. W.; Huang, E. J.; Shen, Y.; Masliah, E.; Mukherjee, C.; Meyers, D.; Cole, P. A.; Ott, M.; Gan, L. Acetylation of tau inhibits its degradation and contributes to tauopathy. Neuron 2010, 67 (6), 953966.

(18) Kontaxi, C.; Piccardo, P.; Gill, A. C. Lysine-directed posttranslational modifications of tau protein in Alzheimer's disease and related Tauopathies. Front. Mol. Biosci. 2017, 4, 56.

(19) Biernat, J.; Gustke, N.; Drewes, G.; Mandelkow, E.; Mandelkow, E. Phosphorylation of Ser262 strongly reduces binding of tau to microtubules: distinction between PHF-like immunoreactivity and microtubule binding. Neuron 1993, 11 (1), 153-163.

(20) Haj-Yahya, M.; Gopinath, P.; Rajasekhar, K.; Mirbaha, H.; Diamond, M. I.; Lashuel, H. A. Site-Specific Hyperphosphorylation Inhibits, Rather than Promotes, Tau Fibrillization, Seeding Capacity, and Its Microtubule Binding. Angew. Chem. 2020, 132 (10), 40884096.

(21) Simic, G.; Babic Leko, M.; Wray, S.; Harrington, C.; Delalle, I.; Jovanov-Milosevic, N.; Bazadona, D.; Buee, L.; de Silva, R.; Di Giovanni, G.; Wischik, C.; Hof, P. Tau protein hyperphosphorylation and aggregation in Alzheimer's disease and other tauopathies, and possible neuroprotective strategies. Biomolecules 2016, 6 (1), 6 .

(22) Lindstedt, P. R.; Taylor, R. J.; Bernardes, G. L. J.; Vendruscolo, M. Facile installation of post-translational modifications on the tau protein via chemical mutagenesis. ACS Chem. Neurosci. 2021, 12 (3), $557-561$.

(23) Song, Y.; DiMaio, F.; Wang, R. Y.-R.; Kim, D.; Miles, C.; Brunette, T.; Thompson, J.; Baker, D. High-resolution comparative modeling with RosettaCM. Structure 2013, 21 (10), 1735-1742.

(24) Lindorff Larsen, K.; Piana, S.; Palmo, K.; Maragakis, P.; Klepeis, J. L.; Dror, R. O.; Shaw, D. E. Improved side chain torsion potentials for the Amber ff99SB protein force field. Proteins: Struct., Funct., Genet. 2010, 78 (8), 1950-1958.

(25) Jorgensen, W. L.; Chandrasekhar, J.; Madura, J. D.; Impey, R. W.; Klein, M. L. Comparison of simple potential functions for simulating liquid water. J. Chem. Phys. 1983, 79 (2), 926-935.
(26) Hess, B.; Bekker, H.; Berendsen, H. J.; Fraaije, J. G. LINCS: a linear constraint solver for molecular simulations. J. Comput. Chem. 1997, 18 (12), 1463-1472.

(27) Bussi, G.; Donadio, D.; Parrinello, M. Canonical sampling through velocity rescaling. J. Chem. Phys. 2007, 126 (1), No. 014101.

(28) Parrinello, M.; Rahman, A. Polymorphic transitions in single crystals: A new molecular dynamics method. J. Appl. Phys. 1981, 52 (12), 7182-7190.

(29) Tribello, G. A.; Bonomi, M.; Branduardi, D.; Camilloni, C.; Bussi, G. PLUMED 2: New feathers for an old bird. Comput. Phys. Commun. 2014, 185 (2), 604-613.

(30) Daura, X.; Gademann, K.; Jaun, B.; Seebach, D.; Van Gunsteren, W. F.; Mark, A. E. Peptide folding: when simulation meets experiment. Angew. Chem., Int. Ed. 1999, 38 (1-2), 236-240.

(31) Humphrey, W.; Dalke, A.; Schulten, K. VMD: visual molecular dynamics. J. Mol. Graphics 1996, 14 (1), 33-38.

(32) Pettersen, E. F.; Goddard, T. D.; Huang, C. C.; Couch, G. S.; Greenblatt, D. M.; Meng, E. C.; Ferrin, T. E. UCSF Chimera-a visualization system for exploratory research and analysis. J. Comput. Chem. 2004, 25 (13), 1605-1612.

(33) Von Bergen, M.; Friedhoff, P.; Biernat, J.; Heberle, J.; Mandelkow, E.-M.; Mandelkow, E. Assembly of $\tau$ protein into Alzheimer paired helical filaments depends on a local sequence motif (306VQIVYK311) forming $\beta$ structure. Proc. Natl. Acad. Sci. U. S. A. 2000, 97 (10), 5129-5134.

(34) Carlomagno, Y.; Chung, D.-e. C.; Yue, M.; Castanedes-Casey, M.; Madden, B. J.; Dunmore, J.; Tong, J.; DeTure, M.; Dickson, D. W.; Petrucelli, L.; Cook, C. An acetylation-phosphorylation switch that regulates tau aggregation propensity and function. J. Biol. Chem. 2017, 292 (37), 15277-15286.

(35) Cohen, T. J.; Guo, J. L.; Hurtado, D. E.; Kwong, L. K.; Mills, I. P.; Trojanowski, J. Q.; Lee, V. M. The acetylation of tau inhibits its function and promotes pathological tau aggregation. Nat. Commun. 2011, 2 (1), 1-9.

(36) Sirota, F. L.; Maurer-Stroh, S.; Eisenhaber, B.; Eisenhaber, F. Single-residue posttranslational modification sites at the $\mathrm{N}$-terminus, C-terminus or in-between: To be or not to be exposed for enzyme access. Proteomics 2015, 15 (14), 2525-2546.

(37) Chooi, K. P.; Galan, S. b. R.; Raj, R.; McCullagh, J.; Mohammed, S.; Jones, L. H.; Davis, B. G. Synthetic phosphorylation of p38 $\alpha$ recapitulates protein kinase activity. J. Am. Chem. Soc. 2014, 136 (5), 1698-1701.

(38) Chalker, J. M.; Lercher, L.; Rose, N. R.; Schofield, C. J.; Davis, B. G. Conversion of Cysteine into Dehydroalanine Enables Access to Synthetic Histones Bearing Diverse Post-Translational Modifications. Angew. Chem. 2012, 124 (8), 1871-1875. 